\section{Cahiers de Narratologie}

Analyse et théorie narratives

39 | 2021

L'héritage de Ricoeur : du récit à l'expérience

\title{
Discours historique et narrativité
}

\section{Philippe Carrard}

\section{OpenEdition}

\section{Journals}

Édition électronique

URL : https://journals.openedition.org/narratologie/12028

DOI : 10.4000/narratologie.12028

ISSN : 1765-307X

Éditeur

LIRCES

\section{Référence électronique}

Philippe Carrard, «Discours historique et narrativité », Cahiers de Narratologie [En ligne], 39 | 2021, mis en ligne le 03 juin 2021, consulté le 21 juillet 2021. URL : http://journals.openedition.org/narratologie/ 12028 ; DOI : https://doi.org/10.4000/narratologie.12028

Ce document a été généré automatiquement le 21 juillet 2021.

Article L.111-1 du Code de la propriété intellectuelle. 


\title{
Discours historique et narrativité
}

\author{
Philippe Carrard
}

\section{Préliminaires}

1 Historiens, philosophes et théoriciens de la littérature ont souvent débattu de l'appartenance de l'historiographie au genre narratif'. D'un côté, Monika Fludernik (1996: 12) a soutenu dans Towards a Natural Narratology que l'historiographie ne relève pas du récit dans la mesure où elle ne s'attache pas à la description de ce que Fludernik appelle «l'expérientialité ", soit à "l'évocation des expériences qu'on peut avoir dans la vie réelle ». L'historiographie, pour Fludernik, consiste en un "simple calibrage des événements » (1996:19), et son incapacité à communiquer ce que les acteurs du passé ont vécu l'exclut de la catégorie du récit. Fludernik a depuis assoupli sa position. Dans sa contribution à un ouvrage consacré précisément au sujet des relations entre l'histoire et l'expérience, elle admet ainsi que les textes historiographiques sont situés sur une "échelle de narrativité ", ce qui entraîne que "plus une étude de ce genre est universitaire, moins elle contient d'expérientialité et donc de narrativité " (Fludernik 2010 : 50). Fludernik prend pour exemples Terror und Traum: Moskau 1937 de Karl Schlögel et 428, une année ordinaire à la fin de l'Empire romain de Giusto Traina, études qui, selon elle, traitent certes de la vie quotidienne, mais ne peuvent être considérées comme des récits parce qu'elles ne communiquent pas "mimétiquement » le "vécu " des individus dont elles parlent (2010:59). Le seul ouvrage de son corpus que Fludernik tient pour appartenir au genre narratif attendu qu'il fait partager «l'expérience des sujets historiques» (2010: 69) est Certitudes meurtrières, accompagnées de quelques spéculations de Simon Schama. Or, notons-le au passage, cet ouvrage a souvent été condamné par la communauté historienne; il s'autorise en effet pour communiquer l'« expérience» des acteurs à utiliser des techniques "littéraires» telles que la focalisation interne, techniques que cette communauté rejette parce qu'incompatibles avec le modèle documentaire sur lequel elle fonde son entreprise.

2 À l'inverse de Fludernik, plusieurs spécialistes des sciences humaines et sociales maintiennent que les historiens, quoi que ceux-ci puissent affirmer dans leurs écrits 
programmatiques, recourent toujours et comme inévitablement au récit lorsqu'ils mettent en texte les données qu'ils ont réunies. En France, cette position a d'abord été défendue par Paul Ricœur, qui - contre les théories avancées par les représentants des Annales et de la Nouvelle Histoire - a soutenu dans Temps et récit que « l'histoire la plus éloignée de la forme narrative continue d'être reliée à la compréhension narrative par un lien de dérivation, que l'on peut reconstruire pas à pas, degré par degré, par une méthode appropriée » $(1983: 133)$. Pour Ricœur, même une étude aussi souvent donnée comme un modèle d'histoire anti-narrative que La Méditerranée et le monde méditerranéen à l'époque de Philippe II de Fernand Braudel est sous-tendue dans sa structure profonde par une "quasi-intrigue virtuelle » : en l'occurrence par un récit qui, dans la «longue durée », se déroule selon le schéma prototypique du "déclin », l'histoire se déplaçant « vers l'Atlantique et l'Europe du Nord » aux dépens de la Méditerranée (Braudel : 300). Quelle qu'ait été leur précédente appartenance doctrinale, plusieurs historiens français ont dans ce domaine adopté la thèse de Ricœur. Roger Chartier (2006: 969), par exemple, affirme maintenant que « l'écriture de l'histoire, même la plus quantitative, même la plus structurale, appartient au genre du récit, dont elle partage les catégories fondamentales ». De même, François Hartog (2005: 165) assure que de "l'histoirerhétorique » de l'Antiquité dont il est le spécialiste à "l'histoire structurale » des Annales, sa discipline " n'a cessé de dire les faits et gestes des hommes, de raconter, non pas le même récit, mais des récits aux formes diverses " (Hartog: 173). En rejetant ce qu'ils appelaient « l'histoire-récit ", Hartog conclut en reprenant la thèse de Ricoeur, les Annalistes n'ont donc pas abandonné le genre narratif; ils en ont évacué « une forme particulière" (Hartog: 172) et inventé de nouvelles, le modèle braudellien constituant à cet égard une innovation non seulement au niveau du contenu, mais à celui de la mise en texte.

3 La thèse selon laquelle les travaux consacrés au passé se présentent nécessairement sous la forme narrative est partagée par nombre de théoriciens anglo-saxons de l'histoire. Alun Munslow (2007: 1), par exemple, un historien britannique connu pour ses positions postmodernes et ses critiques acerbes de l'establishment académique, affirme dès la troisième phrase de sa contribution à l'épistémologie de sa discipline, Narrative and History, que quand les historiens " écrivent ", ils "transforment le 'passé' en ce récit qu'ils choisissent d'appeler 'histoire'». Munslow développe cette affirmation initiale dans des chapitres qu'il intitule "Narrating the Past ", "History as Content/ Story » et "Narrating and Narration » : titres éminemment programmatiques, en ce sens qu'ils excluent implicitement que les travaux historiographiques puissent s'organiser selon d'autres modes que celui du récit. De même, le théoricien néerlandais de l'histoire et spécialiste de Hayden White Herman Paul explique dans Keys Issues in Historical Theory (2015: 57) que les historiens utilisent la "forme narrative ", ou mieux des "schémas [templates] narratifs", pour donner un sens au passé. Ces schémas peuvent correspondre aux types analysés par White dans Metahistory (comédie, tragédie, romanesque, satire), comme ils peuvent prendre la structure de "grands récits" (démocratisation, sécularisation, croissance économique) qui "mettent ordre et cohérence dans le chaos des événements historiques » (Paul : 62). Paul, tout comme Munslow et les historiens français dans la mouvance de Ricœur, semble donc présumer que les historiens recourent toujours au récit. Ceux-ci, quoi qu'ils puissent déclarer dans des interviews ou des textes à caractère théorique, adoptent comme nécessairement la forme narrative lorsqu'ils commencent à " écrire ", soit lorsqu'ils 
passent du stade de la « recherche» au stade de ce que Ricœur (1983: 103) appelle la " configuration », soit de l'agencement des données.

4 Mon essai s'organise en deux temps. Prenant comme corpus un certain nombre d'études universitaires consacrées à la Seconde Guerre mondiale, je pose d'abord la question de savoir si toutes appartiennent au genre narratif. Notant que certaines n'entrent pas dans cette catégorie, j'examine alors celles qui à mon sens en relèvent et m'attache au problème de leur narrativité. Avant de commencer, il me semble toutefois essentiel - ce que les théoriciens qui viennent d'être cités ne font pas toujours - de définir ce que j'entends par " récit ». La théorie littéraire peut sur ce point être utile à l'analyse de l'historiographie, vu qu'un de ses buts est d'établir des distinctions entre les genres discursifs, en l'occurrence de caractériser le récit en le différentiant des autres modes d'organisation textuelle. Adoptant des critères plus formels et plus inclusifs que l'« expérientialité » de Fluderkik, je définirai ici le récit avec Gerald Prince (2012 : 25) comme « la représentation non contradictoire d'au moins deux événements (ou d'un état et d'un événement) asynchrones et se rapportant l'un à l'autre sans se présupposer ou s'impliquer logiquement "; et avec James Phelan (2007 : 203), comme le type de discours où "quelqu'un dit à quelqu'un d'autre, dans certaines circonstances et dans un certain but, que quelque chose s'est passé ». Qu'elles traitent le récit comme une structure ou comme une transaction, ces définitions sont fondées sur la même hypothèse : un texte, pour compter comme récit, doit être constitué d'au moins deux unités situées sur un axe temporel, même si la première de ces unités peut rester implicite. Ainsi, pour prendre des exemples en rapport avec mon corpus, le mini énoncé « Berlin est une ville en Allemagne » n'est pas un récit, parce qu'il ne représente pas d'événement (il ne dit pas que « quelque chose s'est passé»). Mais le minitexte «la ville de Berlin a été prise par les Russes en avril 1945 » est un récit, parce qu'il représente un changement par rapport à un état et pourrait décomposé en «il y a une ville du nom de Berlin » et « cette ville a été prise par les Russes en avril 1945 ».

\section{Analyses synchroniques}

Des sujets tels que « Seconde Guerre mondiale » semblent être intrinsèquement liés à la forme narrative. Une guerre, après tout, n'est-elle pas faite d'événements "asynchrones", que l'historien rapporte "dans certaines circonstances et dans un certain but » aux lecteurs qu'ils pourraient intéresser? En d'autres termes, et pour généraliser, certains sujets n'ont-ils pas en eux-mêmes une structure spécifique, que l'historien se bornera à dégager, puis à reproduire dans le texte qu'il rédige? La question de savoir si les historiens «trouvent» ou «inventent " l'agencement qu'ils confèrent à leurs textes a souvent été débattue, et je n'y reviendrai pas ici². Il me suffira de dire que les études de la Seconde Guerre mondiale qui figurent dans mon corpus tendent à corroborer la thèse selon laquelle l'organisation discursive que l'historien adopte n'émane pas du matériau, mais lui est imposée. Confrontées aux définitions des narratologues, en tout cas, plusieurs de ces études montrent que le sujet "guerre" n'exige pas d'être traité sous la forme du récit. Si, comme tout texte, elles vont d'un point $\mathrm{A}$ à un point $\mathrm{B}$, leurs étapes successives ne sont pas d'ordre temporel, mais 
analytique. La liste qui suit devrait donner une idée très sommaire des sujets dont elles traitent et de la « configuration » qu'elles leur attribuent.

1. Aspects de la guerre. Europe at War, de Norman Davies, envisage diverses facettes du conflit dans des chapitres intitulés respectivement "Interpretation ", "Warfare », Politics ", « Soldiers », « Civilians » et « Portrayals ».

2. Explications. Why the Allies Won, de Richard Overy et Engineers of Victory de Paul Kennedy sont parmi les nombreux ouvrages qui examinent les « causes de ", en l'occurrence du succès des Alliés.

3. Statistiques. La Seconde Guerre mondiale en chiffres de Peter Doyle, est faite de graphiques, de tableaux et de cartes portant sur des sujets qui vont du très général (les armes) au très spécifique (la Bataille d'Angleterre).

4. Controverses. Questions sur la II Guerre Mondiale de Marc Ferro fait le point sur des sujets fréquemment débattus, tels le double jeu du Gouvernement de Vichy, la connaissance de la Shoah (qui savait quoi ?) et l'état d'esprit des populations dans les colonies (Maghreb, Indochine, Inde).

5. Vie quotidienne. Marianne in Chains de Robert Gildea et L'Incendie: l'Allemagne sous les bombes de Jörg Friedrich portent non sur les opérations militaires, mais sur les attitudes des populations civiles face aux épreuves qu'elles subissaient.

6 Si ces études, considérées dans leurs structures globales, ne sauraient être tenues pour des récits, elles n'en sont pas pour autant dénuées d'une dimension narrative. Enchâssé dans l'analyse, un récit comme chez Overy (2009: 208-209) - celui de l'attaque en juin 1944 des tanks allemands par les chasseurs-bombardiers américains Thunderbolt - sert ainsi à montrer la supériorité technologique des Alliés au moment du Débarquement. L'aspect où la période de la guerre que l'historien décrit peut également être considéré comme une phase dans un récit qui peut être prolongé en amont comme en aval. Les nombreux ouvrages consacrés à la condition des populations civiles en France et en Allemagne dans l'immédiat après-guerre sont ainsi susceptibles d'être envisagés, entre autres, comme des suites aux livres de Gildea et de Friedrich. Enfin, et au risque de succomber au pan-narrativisme dont on sait qu'il a connu une certaine vogue, il est loisible d'admettre que certaines analyses de la guerre sont sous-tendues par ce que Ricœur (1983: 298) appelle à propos de La Méditerranée de Braudel une "quasiintrigue", ou encore une "intrigue virtuelle» (1983: 301). Cette intrigue serait susceptible de revêtir différents modèles, à commencer par celui de Propp (1970): allant d'un manque initial à l'élimination de ce manque, elle mènerait des défaites initiales des Alliés à leur victoire finale. Il suffirait, lorsque cela est possible, de replacer dans leur ordre temporel les aspects du conflit que l'historien examine de manière analytique, comme le fait tout lecteur compétent d'un ouvrage de fiction où l'ordre du discours ne correspond pas à celui des événements rapportés.

Dépourvues de narrativité, les études synchroniques de la guerre n'en sont pas pour autant dénuées d'intérêt. Mais cet intérêt ne réside pas dans le développement de l'intrigue, dans la question «Qu'est-ce qui va arriver? »I tient à la nature des faits que l'historien examine et à la manière dont il les présente. Si on leur applique les catégories de Sternberg, des titres tels que Why the Allies Won et Engineers of Victory posent ainsi des lecteurs dont il s'agit d'éveiller la « curiosité » : soit des lecteurs qui ne savent pas pourquoi les Alliés ont gagné la Guerre ou, plus vraisemblablement, qui cherchent à compléter leur connaissance des causes de cette victoire. Mais ces titres impliquent également ce qu'on pourrait appeler un "promesse de surprise»: les 
historiens s'engagent implicitement à fournir des informations inattendues, portant par exemple sur l'identité des «ingénieurs » de la victoire alliée et la nature exacte de leurs travaux. On pourrait dire la même chose d'un titre au premier abord aussi rébarbatif que La Seconde Guerre mondiale en chiffres. Ce titre, en effet, inscrit des lecteurs potentiellement intéressés par les données quantitatives et susceptibles d'être « surpris " par certaines d'entre elles. La question de savoir si les ouvrages qui viennent d'être mentionnés satisfont la "curiosité » de leurs lecteurs réels et ménagent des "surprises» ne peut être résolue dans le cadre d'une analyse textuelle - point sur lequel je reviendrai un certain nombre de fois.

\section{Récits par strates}

8 Si toutes les histoires de la Seconde Guerre mondiale ne relèvent pas du récit, de nombreuses se rangent néanmoins dans cette catégorie discursive. Organisées comme le veulent les définitions de Prince et de Phelan sur un axe temporel, elles peuvent être distribuées en deux classes. La première est celle du récit par strates. Sous cette métaphore géologique, je désigne les études qui découpent le temps en un certain nombre de tranches, qu'elles décrivent et assemblent de manière à constituer une narration. Mais celle-ci n'est pas constituée d'un ou plusieurs événements, à la manière de «la ville de Berlin a été prise par les Russes en avril 1945 ». Elle est faite d'une succession de phases, elles-mêmes souvent formées d'une série d'occurrences groupées sous des désignations très générales telles que " la campagne de France », «l'invasion de la Russie » ou « la guerre dans le Pacifique ».

9 La plupart des histoires de la Seconde Guerre mondiale ressortissent au récit par strates. Elles segmentent le conflit en un certain nombre d'épisodes, ou plutôt de ce que Mikhail Bakhtin (1981: 84-85) appelle des «chronotopes » : elles lient espace et temps, comme le fait par exemple Richard Weinberg quand il intitule des sections de A World at Arms «The Eastern Front and the Changing War: June to December, 1941 », ou "The War at Sea, 1942-1944, and the Blockade ». Or, ces divisions ne sont pas données. Elles résultent de choix que l'historien a effectués, le plus fondamental portant sur la manière de traiter les fronts principaux, soit ceux d'Europe et d'Asie. Weinberg alterne parfois entre les deux, allant par exemple de "The Eastern Front and the Changing War " à "Halting the Japanese Advance " et de "The Final Assault on Germany " à «The War in the Pacific ». Calvocoressi et Wint, en revanche, divisent leur Total War en deux parties principales, consacrées respectivement à «The Western Hemisphere » et « The War in Asia ». L'espace et la durée du chronotope que l'historien assigne à chaque strate varie également de manière considérable. "The Eastern Front from June to December 1941 » constitue ainsi une phase dans le livre de Weinberg, alors que cet espace-temps fait partie d'une strate plus inclusive («Hitler's Wars : 1939-1941») dans l'ouvrage de Calvocoressi et Wint.

10 Tout comme les analyses synchroniques examinées plus haut, les récits par strates peuvent être prolongés en amont comme en aval. Bradley F. Smith, par exemple, soutient que si on l'examine dans la longue durée et la perspective d'une histoire mondiale, le conflit a commencé à l'Est au début des années $1930^{3}$. Il intitule ainsi le premier chapitre de son The War's Long Shadow « China's War ", et il avance que même si les Occidentaux tendent à penser que la Seconde Guerre mondiale a commencé en 1939 ou en 1941, l'invasion japonaise de la Mandchourie en 1931 constitue le premier signe 
d'un conflit plus général. De même, alors que les ouvrages de Weinberg et de Calvocoressi et Wint se terminent sur la défaite du Japon, Smith s'attache aux suites de la guerre. Il examine ainsi les « défis » que les États-Unis, L'URSS, la Chine et la GrandeBretagne ont eu à relever durant la guerre «froide » qui a succédé aux capitulations allemande et japonaise de 1945. Certes, Smith n'affirme pas que la «Seconde Guerre mondiale » a commencé au début des années 1930 et n'était pas terminée en 1950. Mais sa thèse reste claire : le conflit, quelque nom qu'on lui donne, ne saurait être limité aux événements qui se sont déroulés entre 1939 et 1945.

11 Si les récits par strates de la Seconde Guerre mondiale diffèrent quant à la nature qu'ils assignent aux étapes successives du conflit, ils sont en général mis en intrigue de la même manière. J'ai mentionné plus haut que certaines des analyses synchroniques d'un aspect ou d'un moment de la guerre étaient comme sous-tendues par un récit virtuel qui conduisait d'un "manque " à son élimination. Les récits par strates tels que ceux de Weinberg et de Calvocoressi et Wint suivent en gros le même modèle. Dans la terminologie que Hayden White (1973) emprunte à Northrop Frye, ils constituent des romances: des récits dans lesquels le bien finit par triompher du mal, en l'occurrence les Alliés de l'Allemagne et de ses partenaires. Parce qu'il ne s'arrête pas à la fin « officielle » de la guerre mais couvre la période 1945-1950, le livre de Smith offre bien sûr une mise en intrigue différente. Sans devenir ce que White appelle une "satire ", soit un récit dans lequel les activités humaines sont décrites comme dépourvues de signification, il ajoute à la romance un élément d'ironie. Les Alliés, pour Smith, ont certes gagné la guerre ; mais ils n'ont pas atteint les buts qu'ils s'étaient fixés, ou plutôt leurs succès ont eu des conséquences inattendues. Pour citer les titres des derniers chapitres de The War's Long Shadow, l'URSS était ainsi un " walking wounded», la Chine, " a casualty of war », la Grande-Bretagne " permanently disabled », et les États-Unis, " not as healthy, wealthy, and wise » que leurs habitants et le reste du monde ne l'imaginaient.

Les études portant sur la Seconde Guerre mondiale ne sont évidemment pas toutes organisées sur le modèle de la romance. Celles qui traitent de la Shoah, pour prendre l'exemple le plus manifeste, sont habituellement mises en intrigue sous la forme d'une tragédie: d'un récit qui mène à la mort du héros, ici au meurtre de tout un groupe. L'itinéraire d'autres victimes du conflit peut être décrit selon un mode sinon identique, du moins similaire : le récit ne se terminera pas nécessairement sur la mort des acteurs, mais toujours « $\mathrm{mal}$ ». The Deserters de Charles Grass, relate ainsi un parcours par étapes dont les protagonistes vont "from boys to soldiers", "from soldiers to deserters", et finalement «from deserters to military justice». Certains des personnages de ces récits seront condamnés à la peine capitale, alors que d'autres s'en tireront avec des peines de prison.

13 Si les récits par strates sont des récits au sens de Prince et de Phelan, quel est leur exact degré de narrativité? En d'autres termes, si leur structure est celle d'un récit, s'efforcent-ils d'éveiller puis de maintenir l'intérêt du lecteur? Sur ce point, que ni Ricœur, ni les théoriciens mentionnés plus haut (Chartier, Munslow, etc.) n'abordent, il est utile de distinguer avec Prince (2008) entre narrativehood et narrativeness : soit entre « les propriétés qui caractérisent le récit et le distinguent des non-récits » et les " traits optionnels qui font qu'un récit est plus prototypiquement un récit, plus immédiatement identifié, traité et interprété comme un récit». Dans le premier cas, narrativité est une question de «mode discursif : un texte est ou n'est pas un récit »; dans le second, c'est une question de « degré : certains récits sont plus des récits que 
d'autres $»^{4}$. Envisagés dans cette perspective, les récits par strates satisfont aux exigences de la narrativehood : bien que faits de situations, ils ordonnent celles-ci sur un axe temporel. En outre, comme on l'a vu, ils ne constituent pas de simples chroniques qui feraient la liste des événements groupés sous des étiquettes telles que «Halting the Japanese Advance » et "The Final Assault on Germany ». Ils proposent des intrigues qui, selon l'angle sous lequel l'historien a choisi d'aborder son sujet, peuvent prendre des formes allant de la romance à la tragédie.

Mais ces intrigues, si elles confèrent leur cohérence aux données que l'historien a réunies, sont-elles également agencées de manière à retenir l'intérêt du lecteur? Avant de répondre à cette question, il importe, comme on l'a fait à propos de "récit ", de s'entendre sur les termes, en l'occurrence sur le(s) sens conféré(s) à « intrigue ». Selon Karin Kukkonen (2014), une intrigue peut être conçue comme « une structure fixe (...) un modèle qui confère au récit sa cohérence " (Propp); "une structuration... qui reconstruit les pensées de lecteurs qui s'interrogent sur les causes des événements et les mobiles des personnages" (Brooks) ; et « un aspect des intentions de l'auteur (...) des moyens qu'il met en œuvre pour intéresser le lecteur » (Phelan). Quelle que soit la définition adoptée, le danger à mes yeux est de prêter des réactions au lecteur: de présumer par exemple que la "surprise » que l'auteur cherche à provoquer à l'aide de stratégies spécifiques correspond à l'expérience vécue par des lecteurs réels. Seules des études empiriques permettraient de révéler de quelle manière ces lecteurs ont répondu aux invitations de l'auteur, ou du moins de quelle manière ils affirment y avoir répondu. Retenant ici les définitions de l'intrigue comme «structure fixe » et « aspect des intentions de l'auteur", je me demanderai comment, à l'intérieur de cette "structure ", l'« auteur » cherche à éveiller puis à maintenir l'intérêt de son lectorat potentiel.

15 En premier lieu, remarque certes banale, les récits par strates de la Seconde Guerre mondiale ne tentent guère de créer le suspense. Des titres de parties d'ouvrage tels que "The Defeat of Japan" (Calvocoressi et Wint) et "The Final Assault on Germany " (Weinberg) sont à cet égard révélateurs, dans la mesure où ils annoncent immédiatement comment se terminera la phase de la guerre dont va être question. En termes de réception présumée, Total War et $A$ World at Arms posent tous deux un lecteur qui connaît l'issue de cette phase, ou du moins pour qui cette issue (" defeat ", " final assault ») ne vaut pas la peine d'être dissimulée. La narrativeness de récits par strates tels ceux de Weinberg, Calvocoressi et Wint ne dépend donc pas de la manière donc l'historien «suspend » le dénouement de l'intrigue, mais de la manière dont il en lie les différentes étapes, en particulier du moment où il « coupe » pour passer d'une étape à une autre. Baroni (2017: 107-115) consacre plusieurs pages à examiner les «ruptures » qu'introduit la "segmentation" d'un texte en parties ou en chapitres, et bien qu'il s'attache exclusivement à la fiction, les questions qu'il pose sont certainement pertinentes pour l'historiographie. Ainsi, les historiens ressortissent-ils à des cliffhangers? Dans le cas des récits par strates, «coupent-ils» trop tôt, avant que les événements qu'ils rapportent ne soient tout à fait parvenus à leurs termes? La réponse est «oui» et «non». Weinberg (1994: 309), par exemple, termine le chapitre qu'il consacre au Front de l'Est entre juin et décembre 1941 par la phrase « Hitler disait à ses compagnons qu'il préférait une guerre de dix ans à la conclusion de la paix, alors que le Ministre des Affaires étrangères von Ribbentropp, toujours prêt à suivre le Führer, affirmait que l'Allemagne était prête pour une guerre de trente ans ». Comme Weinberg - on l'a vu - pose des lecteurs qui savent comment le conflit s'est terminé, le fait qu'il 
interrompe le récit de ce qui se déroule sur le Front de l'Est pour passer à celui de l'attaque des Etats-Unis par le Japon, ne constitue qu'un demi cliffhanger: la question que pose la coupure n'est pas "la guerre va-t-elle durer plus de dix ans?», mais «quand Weinberg va-t-il revenir au sujet du Front de l'Est? ». La réponse figure déjà dans la table des matières, qui montre que l'historien reprendra le fil de son récit au chapitre XIV, intitulé «The Halt on the European Fronts ». Ainsi, comme Baroni (207 : 111) l'a noté, les cliffhangers qui retardent certaines informations ne sont pas de la même nature dans un roman-feuilleton ou une série télévisée et dans un ouvrage qui se présente immédiatement comme un tout. Dans un roman-feuilleton ou une série télévisée, la suite et/ou la fin de l'histoire ne sont pas disponibles avant le(s) prochain(s) épisode(s). Chez Weinberg, en revanche, comme d'ailleurs en régime fictionnel chez Balzac ou chez Proust, le délai est réduit ; l'information manquante est facilement accessible, le lecteur pressé pouvant même sauter autant de pages, de chapitres, voire de volumes qu'il le veut pour trouver la réponse à la question laissée «suspendue ».

Fondamentalement dénués de suspense, les récits par strates ne sont guère plus riches en effets de surprise. Autrement dit, ils ne cherchent pas à étonner le lecteur en affirmant que le conflit devrait être divisé en moins de phases que les études précédentes ne l'ont fait; que certaines de ces phases mériteraient d'être analysées avec plus de détails qu'elles ne l'ont été dans le passé; que les relations entre le moment $\mathrm{X}$ et le moment $\mathrm{Y}$ de la guerre devraient être réexaminées, etc. Certes, le modèle d'une guerre "longue» qui commencerait dans les années 1930 et se prolongerait jusqu'à la fin des années 1940 est « surprenant » par rapport aux schémas habituels du conflit. Mais il s'agit là d'une question de tellability plutôt que de narrativeness, de contenu plutôt que de stratégie narrative. En d'autres termes, et pour utiliser la définition de Marie-Laure Ryan (2005: 590), c'est le matériau que Smith a choisi de traiter qui est offert comme "défiant les attentes", en l'occurrence celles programmées par la lecture de la plupart des ouvrages sur la Seconde Guerre mondiale. Dans le cas de The War's Long Shadow, c'est donc le projet de Smith plutôt que le récit lui-même qui est censé "surprendre ». Smith n'attend d'ailleurs pas le chapitre IX : « The Beginning of the Cold War in Two Acts : 1945-1946 and 1947-1949 » pour révéler jusqu'à quelle période son examen de la "longue guerre » va se prolonger. Le titre de son livre en annonce déjà le contenu de manière métaphorique, et le dernier paragraphe de son introduction en définit les objectifs : il s'agira de faire en sorte que «la guerre et la guerre froide apparaissent comme participant de la même phase de l'histoire ", restaurant ainsi à cette phase « la place qui lui est propre dans le contexte historique » (Smith $1986: 20$ ).

Dépourvus de suspense et guère agencés pour créer la surprise, les récits par strates n'en sont pas moins susceptibles d'éveiller une au moins des réactions sur la liste de Sternberg, à savoir la curiosité. Dans le cas des ouvrages sur la Seconde Guerre mondiale, le fait que les lecteurs qu'ils inscrivent sachent comment le conflit s'est terminé n'implique pas qu'ils connaissent tous les détails de son déroulement. La curiosité - pour retourner à certains des exemples précédents - peut être éveillée par des engagements pris dans le paratexte, telle la promesse faite par Smith que son ouvrage va restaurer "la place qui lui est propre" à la période qu'il examine. Le lecteur, de manière implicite, est ici invité à se demander comment Smith va conduire sa démonstration, donc à poursuivre au-delà de l'introduction où est cette promesse est effectuée. Mais l'historien qui recourt au récit par strates peut également chercher à 
éveiller la curiosité au moment où, à la fin d'un chapitre ou d'une partie de son ouvrage, il coupe pour passer d'un moment ou d'un lieu du conflit à un autre. Si, on l'a vu, la phrase de Weinberg selon laquelle Hitler disait à ses compagnons qu'il «préférait une guerre de dix ans à la conclusion de la paix » ne génère guère de suspense, elle n'en pose pas moins la question de savoir quels sont les facteurs qui ont abrégé la guerre, rendant ridicule le bluff de Hitler. C'est donc la curiosité qui est censée amener les gens qui s'intéressent à la Seconde Guerre mondiale à entreprendre la lecture d'ouvrages tels ceux de Smith et de Weinberg, puis à la continuer en dépit du nombre souvent considérable des pages qu'ils auront à parcourir - 1178 dans le cas du livre de Weinberg

\section{Récits linéaires}

Si les récits par strates relatent une succession de phases, les récits que je propose d'appeler « linéaires » sont centrés sur un événement ou une série d'événements, dont ils décrivent le déroulement avec autant de détails que les preuves documentaires le permettent. Dans mon corpus, la catégorie "récits linéaires» comprend essentiellement des batailles, un sous-genre dont la popularité peut être facilement testée en entrant des noms tels que «Stalingrad » ou « Iwo Jima » dans le catalogue de n'importe quelle bibliothèque. Je m'occuperai ici du traitement de « Koursk » et de « la Campagne de Normandie ", à savoir de batailles de différentes nature, longueur et importance. Les livres que j'ai choisis, dus à deux spécialistes de l'histoire militaire, sont Koursk: Les quarante jours qui ont ruiné la Wehrmacht (5 juillet-20 août 1943) de Jean Lopez et Decision in Normandy: The Real Story of Montgomery and the Allied Campaign de Carlo D'Este. Après avoir examiné ces études du point de vue de leur structure, je me demanderai quelles stratégies spécifiques leurs auteurs utilisent pour éveiller puis maintenir l'attention du lecteur lorsqu'ils traitent non de toute la guerre, mais d'un de ses moments.

Notons d'abord qu'au niveau de leur agencement global, les ouvrages de Lopez et de d'Este ont en tout cas deux points en commun avec ceux de Weinberg et de Colvocoressi et Wint. Le premier concerne la manière de gérer le récit d'événements simultanés. Qu'ils prennent pour objet une bataille ou le conflit envisagé dans son ensemble, les historiens doivent en effet résoudre le même problème. En raison de la nature linéaire du langage, ils ne peuvent - comme le fait le cartographe -représenter dans un même temps ce ce qui se passe à différents endroits : ils doivent rapporter l'un après l'autre des événements qui, en gros, ont eu lieu au même moment. La bataille de Kourks, par exemple, s'est déroulée sur deux fronts. Enfonçant les lignes ennemies, les Russes avaient créé un saillant que les Allemands attaquaient à partir du nord et du sud. Lopez, comme Colvocoressi et Wint le font à une échelle plus large, choisit de séparer nettement les deux aspects de la bataille, traitant d'abord du Front nord, puis du Front sud. D'Este procède de manière différente. Dans le chapitre qu'il consacre aux événements du 6 juin («D-Day: The Invasion of France»), il passe fréquemment d'un des endroits du débarquement à un autre, rendant compte de ce qui se déroule sur les différentes plages dont les troupes britanniques, américaines et canadiennes tentent de s'emparer. Il décrit la suite de la Campagne selon le même principe, relatant en alternance les opérations conduites par les différents groupes d'armée en marche vers la frontière allemande. 
20 Le second point commun entre les récits qui, dans mon corpus, traitent de la guerre envisagée dans son ensemble et ceux qui s'attachent à une bataille, concerne la structure de l'intrigue. Celle-ci, dans Koursk comme dans Decision in Normandy, est en effet du même type que dans Total War et A World at Arms. Partant d'un manque, elle conduit à son élimination: attaqué, le saillant de Koursk est défendu avec succès ; hautement fortifiées, les falaises de Normandie sont néanmoins conquises. Comme la plupart des études qui traitent de la Seconde Guerre mondiale, les livres de Lopez et de D'Este peuvent donc être considérés comme des romances: le bien finit par triompher du mal, ici les Russes des Allemands à Koursk, les Britanniques, les Américains et les Canadiens, du même adversaire en Normandie. Dans ce dernier cas, le récit de D'Este n'est toutefois pas dépourvu d'un élément «satirique ». Comme Smith le fait dans son examen des conséquences de la guerre, D'Este souligne que les événements ne se sont pas toujours déroulés comme ils auraient dû. Les Britanniques, notamment, ont rencontré à Villars-Bocage une résistance plus vive qu'ils ne l'attendaient, péripétie qui a retardé considérablement la prise de la ville stratégiquement importante de Caen.

21 Bien évidemment tellable pour les amateurs d'histoire militaire, la bataille de Koursk et la Campagne de Normandie sont-elles racontées selon des techniques qui leur confèreraient un certain degré de narrativeness? En d'autres termes, sont-elles décrites non seulement avec une précision érudite, mais - dans la terminologie de Baroni - avec des moyens qui en rendrait le récit "intrigant " ? À nouveau, il convient de souligner qu'il n'y a pas de rapport nécessaire entre tellability et narrativeness. Doyle tient ainsi Koursk et le Débarquement pour des épisodes qui méritent de figurer dans sa Seconde Guerre mondiale en chiffres. Mais ses descriptions prennent la forme de tableaux statistiques portant sur des objets tels que le nombre des tanks et la taille des plages, soit d'un type de discours dont l'intérêt ne dépend pas de l'agencement d'une intrigue. Quant aux théoriciens de l'historiographie tels White (1973) et Mink (2001), ils soutiendraient probablement que comme les intrigues ne sont pas trouvées dans les documents mais construites, aucun événement - aussi tellable semble-t-il - n'est en luimême structuré d'une manière qui en rendrait la relation à coup sûr captivante.

Posant comme la plupart des spécialistes de la Seconde Guerre mondiale un lecteur informé, ni Lopez ni D'Este ne cherchent à générer des effets de suspense qui porteraient sur l'issue des opérations qu'ils rapportent. Lopez est à cet égard particulièrement explicite: le titre qu'il donne à son étude, "Koursk», est immédiatement suivi du sous-titre « Les quarante jours qui ont ruiné la Wehrmach (5 juillet-20 août 1943) ", le paratexte ne laissant aucun doute sur la manière dont la bataille s'est terminée et les conséquences qu'elle a eues. Le titre de D’Este (« Decision in Normandy") et son sous-titre ("The Real Story of Montgomery and the Allied Campaign ») ne sont pas aussi explicites, ou plutôt ils déplacent l'accent de l'issue de la campagne sur le rôle d'un individu. Le texte qui figure en quatrième de couverture fournit toutefois comme un script du déroulement des opérations, informant le lecteur (ou lui rappelant) que cette "campagne sanglante» a non seulement "libéré la France ", mais mené à la « chute de l'Allemagne ».

23 Renonçant au suspense, Lopez et D'Este adoptent d'autres stratégies pour susciter l'intérêt du lecteur. Deux d'entre elles semblent avoir leur préférence. La première consiste à recourir à la prolepse, en privilégiant le type qui pour Baroni $(2017: 14)$ " crée un effet de prédestination ». Examinant le rôle des tanks à Koursk, Lopez (2011 : 65) affirme ainsi que même si «les forces blindées soviétiques n'ont jamais été à la 
hauteur de celles de l'ennemi (...) ce sont elles qui in fine décideront de la victoire à Koursk (...)»; que, parmi les tanks russes engagés dans la bataille, les vingt-et-un exemplaires du SU-152 produits par la fabrique Kirov «seront les seuls à détruire Tigres, Panthers et Ferdinands à plus de 1000 mètres " (2011:68); et qu'un des épisodes décisifs de la bataille « se jouera au sud (...) dans un quadrilataire de $30 \mathrm{~km}$. de hauteur sur $50 \mathrm{~km}$. de base, un mouchoir de poche à l'échelle du front de l'est » (2011: 81). De même, faisant allusion aux « controverses » et aux «mythes » qu'il passera en revue aux chapitres XXVI à XXVIII de son étude, D'Este (2004:119) écrit à la fin du chapitre VII que « les hommes de la Troisième Division britannique » ne soupçonnaient pas que leurs actions "seraient l'objet de fréquents débats dans les années à venir "; que du Débarquement à la fin de la guerre, le problème du manque d'infanterie de renfort bien entraînée "allait préoccuper Montgomery" (2004: 255); et que "personne ne savait que les semaines de frustration et d'inquiétude qui avaient suivi le 6 juin allaient se terminer avec COBRA » (2004: 400), la percée américaine qui avait commencé le 25 juillet. La fonction des prolepses est ici clairement d'éveiller la curiosité du lecteur en rompant la continuité d'un récit hautement linéaire. Annonçant certains des événements à venir, les prolepses creusent un "trou " que l'historien comblera lorsqu'il reviendra à des sujets tels que les performances du tank soviétique SU-152 ou le manque de fantassins bien entraînés dans l'armée britannique. Les prolepses, en outre, sont parfois situées à des endroits stratégiques, comme c'est le cas de la référence à COBRA, placée à la fin du chapitre qui précède le récit de cette opération. D'Este recourt ici à un cliffhanger, il est vrai guère générateur de suspense. La question qu'il soulève concerne en effet le «comment" plutôt que le "quoi", le déroulement des événements plutôt que leur issue - que la prolepse a déjà dévoilée.

La seconde stratégie que Lopez et D'Este déploient pour maintenir l'intérêt du lecteur consiste à introduire des hypothèses contrefactuelles, soit à poser la question " et si ? ». L'histoire contrefactuelle est un genre populaire chez les historiens et les spécialistes des sciences politiques (Tetlock et Belkin 1996, Ferguson 1997, Cowley 2001), toujours prêts à «rejouer» des événements tels que la bataille de Waterloo, les accords de Munich ou la crise des missiles cubains ${ }^{5}$. Les ouvrages de Lopez et de D'Este ne relèvent certainement pas de ce type d'histoire, mais tous deux incluent parfois ce que Quentin Deluermoz et Pierre Singaravélou (2016: 24) appellent des «digressions contrefactuelles ", soit des passages où l'historien examine brièvement «ce qui serait advenu, si ». Cette manière de considérer des alternatives au passé réel se présente sous deux formes principales. La première consiste en une hypothèse faite au conditionnel, suivi d'un énoncé (qui peut rester virtuel) expliquant que cette hypothèse n'a pas été actualisée : "À l'évidence, Manstein aurait dû saisir depuis longtemps les hauteurs situées face au XXXVII e Panzerkorps. Son attaque aurait gagné en percussion et les Soviétiques n'auraient découvert le Schwerpunkt qu'au moment même de l'assaut. Hitler et l'OKH ont interdit cette opération en juin (...) » (Lopez $2011: 151)$; «S'emparer de Noyers et d'Evrecy aurait permis de précariser la situation de la $12^{\mathrm{e}}$ Division SS, prenant cette dernière en tenaille entre la $3^{e}$ Division canadienne, la $7^{\mathrm{e}}$ Division blindée et d'autres unités du 30 Corps" (D'Este $2004: 200$ ). Le second mode de digression contrefactuelle consiste en une proposition introduite par « si » qui pose une condition, une proposition au conditionnel qui énonce ce qui serait arrivé si cette condition avait été remplie, puis une proposition à l'indicatif (qui peut elle aussi rester virtuelle) décrivant ce qui s'est effectivement passé : «Cette action latérale aurait pu, si elle avait réussi, avoir des conséquences importantes sur le déroulement de la bataille » (Lopez 
2011 : 166) ; «Si les Allemands, dans les jours qui ont suivi le Débarquement, avaient choisi de renforcer leurs forces en Normandie avec des division de la Quinzième Armée et les réserves de l'OKW avant que Montgomery ait disposé de toutes ses troupes, ils auraient pu inverser le cours de la bataille » (D'Este 2004 : 159). Au même titre que les prolepses, les énoncés contrefactuels brisent la continuité du récit. Ils ne le font toutefois pas en annonçant ce qui va arriver, mais en soulignant ce qui aurait pu arriver si telle ou telle condition avait été remplie. Lorsqu'ils formulent une hypothèse qu'ils rejettent ou "dénarrent" (Prince 1988), les historiens - pour recourir à la célèbre métaphore proposée par Borges - signalent qu'ils sont conscients du fait que le passé a comporté de nombreuses "fourches", soit de moments où les choses ont "bifurqué " dans une direction alors qu'elles auraient aller dans une autre. Ces pauses contrefactuelles invitent le lecteur à se poser des questions, en l'occurrence à suivre l'historien lorsque celui-ci interrompt brièvement son récit pour examiner le rôle que la contingence a pu jouer dans le passé, à expliquer comment un événement aurait pu se dérouler autrement qu'il ne l'a fait.

Si Lopez et D'Este recourent à des prolepses et à des hypothèses contrefactuelles pour retenir l'intérêt du lecteur, ils ne cherchent guère à ménager des effets de surprise. $\mathrm{Ou}$ plutôt, ils réservent ces effets pour les sections de leurs études où ils argumentent plus qu'ils ne racontent. Lopez consacre ainsi le chapitre II de la cinquième partie de son livre à déboulonner les "idées reçues " (titre du chapitre) sur Koursk, notamment les thèses selon lesquelles "Hitler a gâché l'opération par son indécision » et Koursk a constitué «le chant du cygne des Panzers». Plus direct encore, D'Este affirme immédiatement son ambition de nouveauté lorsqu'il donne à son ouvrage le sous-titre The Real Story of Montgomery and the Allied Campaign (dans une autre édition, le sous-titre proclame : The Unwritten Story of [...]). D'Este promet ici explicitement que son livre va fournir des informations inédites, voire même, corrigeant certaines versions erronées, la «bonne » version de la Campagne de Normandie. La thèse de D'Este est que cette Campagne ne s'est pas déroulée comme elle aurait dû, notamment que les plans de Montgomery n'ont pu être exécutés, ce qui a retardé l'avance alliée de plusieurs semaines. D'Este défend ces vues dans l'ensemble de son ouvrage, et il y revient dans la section finale ("Aftermaths») où il dénonce ce qu'il appelle le «mythe de la Normandie ». Des expressions telles que «idées reçues » et "mythe de la Normandie » sont bien sûr censées éveiller la curiosité. Mais elles comportent aussi la promesse d'une surprise: l'historien assure que son ouvrage va apporter quelque chose non seulement de nouveau, mais d'inattendu. Lopez et D'Este mettent ici en clair ce qui chez d'autres historiens reste souvent implicite, à savoir que toute étude historique, quelque originale qu'elle se prétende, est nécessairement réécriture, en ce sens qu'elle renvoie à d'autres études, qu'elle reprend, critique et/ou transforme.

\section{Le propre de l'historiographie}

Les procédés auxquels Lopez et D'Este recourent pour susciter l'intérêt du lecteur ne sont pas particuliers à ces historiens. La plupart des travaux historiographiques incluent des hypothèses contrefactuelles et affirment apporter des informations inédites. Comme Dorrit Cohn (1999) parle du "propre de la fiction", on pourrait dire que ces dispositifs textuels participent du " propre de l'historiographie $»^{6}$. La présence $d^{\prime}$ 'hypothèses contrefactuelles est en effet difficilement compatible avec les conventions 
du discours fictionnel, du moins dans la situation narrative qui est celle de Lopez, de D'Este et de la plupart des historiens : un narrateur extra- et hétérodiégétique rend compte d'événements auxquels il n'a pas pris part et dont dans la plupart des cas il n'a même pas été le témoin. Certes, Hilary Dannenberg (2008:123) a trouvé des exemples d'énoncés contrefactuels dans des romans anglais dont le narrateur est précisément de ce type, notamment dans Mansfield Park de Jane Austen. Dannenberg n'explique pas si cet usage est caractéristique de la variante britannique de ce qu'il est convenu d'appeler le roman «classique " du dix-neuvième siècle ou si on peut en trouver des occurrences chez les écrivains "continentaux». Il est toutefois difficile d'imaginer Flaubert, par exemple, mettre au compte de son narrateur des hypothèses du type «Emma aurait pu décider de ne pas prendre le poison, mais... » ou «Si Emma n'avait pas eu de liaison avec Rodolphe, alors...». De telles conjectures, en régime hétérodiégétique, sont conventionnellement réservées aux personnages, dans l'esprit desquels elles prennent la forme de ce que Marie-Laure Ryan (1991:156) appelle des "récits virtuels enchâssés ». Mais elles peuvent être assignées sans problème au narrateur en régime homodiégétique, c'est-à-dire dans les récits où le narrateur est l'un des personnages et, en tant que tel, est libre de spéculer sur «ce qui aurait pu arriver, si... ".

Pour des philosophes tels Avezier Tucker, la fréquente occurrence d'hypothèses contrefactuelles en histoire est liée à la nature des explications dans cette discipline. Il serait en effet difficile, pour un historien, d'expliquer pourquoi les événements se sont déroulés d'une certaine manière sans considérer les alternatives. Recourir au raisonnement contrefactuel permet de prendre mesure de la contingence, et de distinguer la contingence de la nécessité. Tucker (2004:239) insiste sur le fait que cette démarche doit rester soumise aux "exigences de la documentation ", soit aux règles sur l'établissement de la preuve qui sont de mise dans la communauté historienne. Lopez comme D'Este restent à cet égard d'une extrême prudence : leurs spéculations ne portent que sur Koursk et la Normandie, D'Este refusant par exemple-au contraire de nombre de ses collègues-de s'interroger sur les conséquences qu'aurait pu avoir sur la Campagne, s'il avait réussi, l'attentat contre Hitler du 20 juin 1944.

Tout comme le recours aux hypothèses contrefactuelles, la mise en question de certains aspects des versions précédentes d'un événement semble être propre à l'historiographie. Si Lopez et D'Este peuvent sans problème dénoncer ce qu'ils appellent les «mythes » associés à la bataille de Koursk et à la campagne de Normandie, on voit mal Flaubert - pour revenir une fois encore aux conventions du roman du dixneuvième siècle - assigner à son narrateur la tâche d'examiner de manière critique les relations préalables de la mort d'Emma avant de proposer la sienne. Comme le recours au raisonnement contrefactuel, ces activités de comparaison et d'évaluation sont dans un récit extra- et hétérodiégétique réservées aux personnages. Elles peuvent en revanche s'exercer sans problème dans un récit homodiégétique, où il est commun que le narrateur présente les versions rivales d'un événement, puis explique pourquoi l'une lui a semblé meilleure que les autres, ou encore pourquoi, aucune n'étant satisfaisante, il a été conduit à élaborer la sienne propre. Au même titre que les hypothèses contrefactuelles, ces références critiques aux travaux précédents ont une fonction précise en historiographie. De même qu'il est difficile d'expliquer pourquoi certains événements du passé se sont déroulés d'une manière et pas d'une autre sans imaginer des alternatives, de même il est difficile d'affirmer que les informations fournies dans 
une étude sont nouvelles sans renvoyer à d'autres études, où ces informations sont absentes ou présentées sous une forme jugée insatisfaisante.

J'ai avancé plus haut qu'une des fonctions des titres et des sous-titres que Lopez et D'Este (ou leurs éditeurs) avaient choisis pour leurs ouvrages était d'attiser l'intérêt du lecteur en faisant des promesses, en l'occurrence en s'engageant à livrer des informations inédites et à dissiper les idées reçues. Lorsqu'ils mettent en doute que Koursk ait vu la plus grande bataille de chars de la Seconde Guerre mondiale ou que la campagne de Normandie se soit déroulée selon les plans de Montgomery, Lopez et D'Este s'exécutent: ils montrent qu'ils ont tenu parole, en ce sens qu'ils ont été capables de bousculer certains mythes et de décrire avec exactitude "ce qui s'était vraiment passé ». Qu'ils l'aient fait aux dépens d'ouvrages jugés fiables tels les mémoires de Montgomery peut ajouter à la rentabilité leur démarche, selon le principe " plus le texte mis en question est dû à un personnage connu et jugé digne de confiance, plus grande devrait être la surprise ». Je dis « devrait " parce que - répétons-le - les réactions des lecteurs réels de Koursk et de Decision in Normandy aux hypothèses contrefactuelles et informations supposément inédites que contiennent ces deux ouvrages ne peuvent être décrites au moyen d'une analyse textuelle. Cette dernière ne permet de déterminer ni si ces lecteurs ont été authentiquement surpris par les informations qu'ils découvraient, ni même s'ils ont été assez curieux pour aller jusqu'à la fin de livres fort longs et à l'intrigue guère captivante, parce que connue.

\section{BIBLIOGRAPHIE}

Bakhtin, Mikhail (1981 [1937]), «Forms of Time and of the Chronotope in the Novel », dans The Dialogic Imagination : Four Essays, trad. Michael Holquist, Austin, University of Texas Press, p. 84-258.

Baroni, Raphaël (2017), Les Rouages de l'intrigue : Les outils de la narratologie postclassique pour l'analyse des textes littéraires, Genève, Slatkine Érudition.

Braudel, Fernand (1966), La Méditerranéee et le monde méditerranéen à l'époque de Philippe II, Paris, Armand Colin.

Calvocoressi, Peter, et Guy Wint (1989), Total War : The Causes and Courses of the Second World War, Londres, A. Lane.

Chartier, Roger (2006), « Récit et histoire », dans Sylvie Mesure et Patrick Savidan, dir., Le Dictionnaire des sciences humaines, Paris, PUF, p. 969-972.

Cohn, Dorrit (2001), Le Propre de la fiction, trad. Claude Hary-Schaeffer, Paris, Seuil.

Cowley, Robert, dir. (1999), What if? The World's Foremost Military Historians Imagine What Might Have Been, New York, G.P. Putnam's Sons.

Dannenberg, Hilary (2008), Coincidence and Counterfactuality: Plotting Time and Space in Fiction, Lincoln, University of Nebraska Press.

Davies, Norman (2006), Europe at War, 1939-1945 : No Simple Victories, Londres, Macmillan. 
Deluermoz, Quentin, et Pierre Singaravélou (2016), Pour une histoire des possibles : Analyses contrefactuelles et futurs non advenus, Paris, Seuil.

D'Este, Carlo (2004), Decision in Normandy : The Real Story of Montgomery and the Allied Campaign, London, Penguin Books.

Doyle, Peter (2013), La Seconde Guerre mondiale en chiffres, trad. Antonia Leibovici, Paris, ContreDires.

Dray, William H. (2001), « Narrative and Historical Realism », dans Geoffrey Roberts, dir., The History and Narrative Reader, New York, Routledge, p. 157-180.

Ferguson, Niall, dir. (1997), Virtual History : Alternatives and Counterfactuals, Londres, Picador.

Ferro, Marc (1993), Questions sur la II ${ }^{e}$ Guerre mondiale, Paris, Casterman.

Fludernik, Monika (1966), Towards a "Natural" Narratology, New York, Routledge.

Fludernik, Monika (2010), « Experience, Experientiality, and Historical Narrative: A View from Narratology », dans Thiemo Breyer et Daniel Cruz, dir., Erfahrung und Geschichte: Historische Sinnbildung im Pränarrativen, Berlin / Boston, De Gruyter, p. 40-72.

Friedrich, Jörg (2004), L'Incendie : L'Allemagne sous les bombes, 1940-1945, trad. Isabelle Hausser, Paris, de Fallois.

Gallagher, Catherine (2018), Telling It Like It Wasn't : The Counterfactual Imagination in History and Fiction, Chicago, University of Chicago Press.

Gildea, Robert (2003), Marianne in Chains : Daily Life in the Heart of France During the German Occupation, New York, Metropolitan Books.

Grass, Charles (2013), The Deserters : A Hidden History of World War Two, New York, Penguin.

Hartog, François (2005), Évidence de l'histoire : Ce que voient les historiens, Paris, Editions de l'EHESS.

Kennedy, Paul (2013), Engineers of Victory: The Problems Solvers Who Turned the Tide in the Second World War, London, Allen Lane.

Kukkonen, Karen (2014), « Plot », dans The Living Handbook of Narratology, www.lhn.unihamburg.de/article/plot, consulté le 9/12/2020.

Lopez, Jean (2011), Koursk : Les quarante jours qui ont ruiné la Wehrmacht (5 juillet-20 août 1943), Paris, Economica.

Mink, Louis (2001 [1978]), « Narrative Form as a Cognitive Instrument », dans Geoffrey Roberts, dir., The History and Narrative Reader, New York, Routledge, p. 211-220.

Munslow, Alun (2007), Narrative and History, New York, Palgrave Macmillan.

Overy, Richard (1995), Why the Allies Won, New York, Norton.

Paul, Herman (2015), Key Issues in Historical Theory, New York, Routledge.

Phelan, James (2007), Experiencing Fiction: Judgments, Progressions, and the Rhetorical Theory of Narrative, Columbus, Ohio State University Press.

Prince, Gerald (1988), « The Disnarrated», Style 22, 1, p. 1-8.

Prince, Gerald (2008), « Narrativehood, Narrativeness, Narrativity, Narratability », dans John Pier et Angel Garcia Landa, dir., Theorizing Narrativity, Berlin/Boston, De Gruyter, p. 19-27.

Prince, Gerald (2012, « Récit minimal et narrativité », dans Sabrinelle Bedrane, Françoise Revaz et Michel Viegnes, dir., Le Récit minimal, Paris, Presses de la Sorbonne nouvelle, p. 23-32. 
Propp, Vladimir (1970), Morphologie du conte, trad. Claude Ligny, Paris, Gallimard.

Ricœur, Paul (1983), Temps et récit, tome I, Paris, Seuil.

Ryan, Marie-Laure (1991), Possible Worlds, Artificial Intelligence, and Narrative Theory, Bloomington, Indiana University Press.

Ryan, Marie-Laure (2005), « Tellability », dans David Herman, Manfred Jahn et Marie-Laure Ryan, dir., Routledge Encyclopedia of Narrative Theory, New York, Routledge, p. 589-591.

Schama, Simon (1996), Certitudes meurtrières, accompagnées de quelques spéculations, trad. Josée Kamoun, Paris, Seuil.

Schlögel, Karl (2008), Terror und Traum: Moskau 1937, Munich, Hanser.

Smith, Bradley F. (1986), The War's Long Shadow: The Second World War and Its Aftermath, China, Russia, Britain, America, New York, Simon and Schuster.

Sternberg, Meir (1978), Expositional Modes and Temporal Ordering in Fiction, Baltimore, Johns Hopkins University Press.

Tetlock, Philip E., et Aaron Belkin, dir. (1996), Counterfactual Thought Experiments in World Politics : Logical, Methodological, and Psychological Perspectives, Princeton, Princeton University Press.

Traina, Giusto (2009), 428 : Une année ordinaire à la fin de l'Empire romain, Paris, Les Belles Lettres.

Tucker, Avezier (2004), Our Knowledge of the Past : A Philosophy of Historiography. Cambridge, Cambridge University Press.

Weinberg, Gerhard (1994), A World at Arms : A Global History of World War II, Cambridge, Cambridge University Press.

White, Hayden (1973), Metahistory: The Historical Imagination in Nineteenth-Century Europe, Baltimore, Johns Hopkins University Press.

\section{NOTES}

1. C'est en toute connaissance de cause que je renonce ici à utiliser l'écriture dite "inclusive ", qui me semble difficile à appliquer de manière conséquente.

2. Pour un résumé des débats entre « réalistes » (Carr) et « constructivistes » (Mink, White), voir par exemple Dray 2001.

3. Spécialiste de la Seconde Guerre mondiale, Bradley R. Smith ne doit pas être confondu avec Bradley R. Smith, auteur de Confessions of a Holocaust revisionist et membre d'organisations négationnistes telles que l'Institute for Historical Review et le Committee for Open Debate on the Holocaust.

4. Dans une conférence prononcée à Québec mais jamais publiée, Prince a proposé les équivalents français «narrativauté » et " narrativitude » à «narrativehood» et «narrativeness " (courriel de Prince du 20 novembre 2020). Ces néologismes n'étant pas (encore) entrés dans l'usage, je conserverai ici l'anglais.

5. Le genre, bien sûr, est également pratiqué par les romanciers. Sur ce sujet, voir par exemple Gallagher 2018.

6. Le titre original de Cohn est The Distinction of Fiction, « distinction » étant pris ici dans son sens étymologique de « différence ». 


\section{RÉSUMÉS}

La plupart des chercheurs qui s'intéressent aux questions d'écriture en sciences humaines et sociales semblent aujourd'hui tenir le genre narratif pour celui que les historiens ne peuvent éviter lorsqu'ils mettent en texte les données qu'ils ont réunies. Or, l'examen d'un corpus constitué de plusieurs histoires de la Seconde Guerre mondiale montre que certaines de ces études ne relèvent pas du récit, du moins tel que le genre est défini par les narratologues. Celles qui peuvent être considérés comme des récits, d'autre part, se présentent sous différentes formes et offrent différents degrés de narrativité. Si, posant un lecteur qui sait comment le conflit s'est terminés (par la victoire des Alliés), elles renoncent au suspense, elles cherchent en revanche à éveiller la curiosité en formulant des hypothèses contrefactuelles ( «Et si... »), de même qu'à créer la surprise en proposant des «versions inédites de » basées sur la découverte de nouveaux documents ou l'introduction de nouvelles questions. Comme Dorrit Cohn parle du «propre de la fiction », on pourrait dans ce domaine parler du « propre de l'historiographie ». Les récits extraet hétérodiégétiques dits "classiques» auxquels les études historiques sont souvent comparées ne sont en effet susceptibles ni de contenir des hypothèses contrefactuelles, ni d'offrir des événements qu'ils rapportent une nouvelle version " plus conformes aux faits ».

\section{INDEX}

Index chronologique : 20e-21e siècle

Mots-clés : discours historique, narrativité, analyse synchronique, récit par strates, récit linéaire, hypothèse contrefactuelle, nouvelle version de

Index géographique : monde occidental

\section{AUTEUR}

\section{PHILIPPE CARRARD}

Dartmouth College et University of Vermont 